\title{
Releer a Daniel Bell. A cuarenta años de la publicación de Las contradicciones culturales del capitalismo
}

\section{To reread to Daniel Bell. Forty years after the publication of The Cultural Contradictions of Capitalism}

doi: http://dx.doi.org/10.32870/

espiral.v24i68.5741

Jorge Ramírez Plascencia*

Héctor Raúl Solís Gadea••

\begin{abstract}
Resumen
Las contradicciones culturales del capitalismo, publicado por Daniel Bell en 1976, ejerció una visible influencia en los debates intelectuales de esos años y ha mantenido una presencia relativamente constante en la literatura sociológica. Bell sostuvo principalmente que la cultura había cambiado radicalmente en una dirección que la hacía incompatible con la reproducción de la economía capitalista. A cuarenta años de la aparición del libro, en este artículo se busca valorar la consistencia de esta tesis. La perspectiva asumida es crítica. Se pretende mostrar sus debilidades revelando el uso inmoderado de la idea de Weber sobre la relación entre ética protestante y desarrollo del capitalismo, las deficiencias de la teoría de la sociedad que construye Bell para pensar la existencia de contradicciones y la adopción de conceptos de cultura y religión demasiado restrictivos.
\end{abstract}

Palabras clave: capitalismo, Daniel Bell, modernismo, contradicciones culturales, teoría social.

\begin{abstract}
The Cultural Contradictions of Capitalism, published by Daniel Bell in 1976, exercised a visible influence on the intellectual debates of those years and it has maintained a relatively constant presence in sociological literature. Bell argued mainly that culture had radically changed in a direction that made it incompatible with the reproduction of the capitalist economy. Forty years after the appearance of the book, in this article we seek to assess the consistency of this thesis. The perspective is critic. We want to show its weaknesses revealing the misuse of Weber's idea concerning the relationship between protestant ethic and development of capitalism, the shortcomings of the theory of society built by Bell in order to settle the existence of contradictions and the adoption of concepts of culture and religion restrictives.
\end{abstract}

Keywords: capitalism, Daniel Bell, modernism, cultural contradictions, social theory.

\footnotetext{
- Profesor-Investigador del Centro Universitario de Ciencias Sociales y Humanidades (CUCSH), Universidad de Guadalajara, México._joraplas@gmail.com

- Profesor-Investigador del Centro Universitario de Ciencias Sociales y Humanidades (CUCSH), Universidad de Guadalajara, México. raulso@gmail.com

Fecha de recepción: 24 de junio de 2016. Fecha de aceptación: 06 julio de 2016.
} 


\section{Introducción'}

Aparecido en 1976, Las contradicciones culturales del capitalismo (en adelante Las contradicciones) es considerado uno de los libros más influyentes de la segunda mitad del siglo $\mathrm{XX}^{2} \mathrm{y}$ su presencia ha sido constante en el análisis y debate académicos (véanse, como ejemplos: Featherstone, 1991; Illouz, 1992; Yates y Hunter, 2011).

Las contradicciones es un libro polémico y arriesgado, objeto de numerosos comentarios y de duras críticas desde la publicación inicial de su principal tesis, en 1970 (Bensman y Vidich, 1972; Crittenden, 1972; Muñiz, 1978; Phillips, 1978; Habermas, 1983). A medio camino entre el análisis y la crítica, entre la neutralidad científica y el compromiso ideológico, es un libro singular entre los que aparecieron entonces y que también pretendían hacer un diagnóstico de esa época, como el de Touraine (1971) y Crozier, et al. (1975), Habermas (1991) y Offe (1990).

A diferencia de ellos, cuyo enfoque es más analítico y su alcance global, el trabajo de Bell es más idiosincrático y está más centrado en los problemas de la sociedad estadounidense de ese momento. En sus páginas se observa la preocupación por el fin del largo periodo de bonanza económica que se registró a nivel mundial entre 1950 y 1970, conocido entre los economistas como la "edad dorada" (Cairncross y Cairncross, 1992; Marglin y Schor, 1992), así como también la inquietud por los cambios en el arte, la cultura y la vida cotidiana estadounidenses (entre otros, la emergencia del arte pop, la liberación sexual, la cultura

I. Este artículo presenta resultados del proyecto "Sobre la actualidad y heurística de la teoría social a través de algunos de sus autores", del cuerpo académico UdeG-CA-405, que contó con recursos financieros PROMEP/SEP, Fortalecimiento de CA, Convocatoria 2014.

2. Según el Times Literary Supplement en su edición del 06 de octubre de 1995. 
hippie, los movimientos de protesta y la aparición de una sociedad de consumo). ${ }^{3}$

La lectura y análisis de Las contradicciones presenta siempre un desafío. Hay que tener en cuenta que se trata de un libro compuesto por trabajos que se publicaron con anterioridad y que Daniel Bell reunió tratando de que formaran una narrativa coherente (como lo indica él mismo en la parte de "Reconocimientos"). No lo logró del todo y el libro presenta, irónicamente, algunas contradicciones y argumentos inconclusos.

Pero también se debe considerar que es un libro que no se cerró con su publicación inicial en 1976, sino que alrededor de una cuarta parte de su versión definitiva proviene de dos añadidos posteriores: del prefacio de 1978, y del epílogo de 1996 que apareció con motivo del vigésimo aniversario de su edición original. Estos agregados surgieron seguramente aconsejados por la necesidad de clarificar las ideas originales y de reconocer, aunque de modo tácito, algunos de sus problemas.

Sin embargo, el reto mayor para su análisis proviene de su composición: una sutil mezcla de análisis sociológico, crítica cultural, retórica conservadora y programa político, todo a la vez. Cada uno de estos hilos llevaría el análisis por caminos que obligan a revisar amplias constelaciones intelectuales y culturales de la mitad del siglo pasado. Estas constelaciones estructuran la peculiar visión que expresa Daniel Bell en ese libro. Se podría enmarcar su crítica del modernismo, por ejemplo, como parte de una actitud antimodernista más amplia de la intelectualidad judía (MacDonald, 1998). También podría revisarse en qué medida la posición política e ideológica de Bell formó parte y ayudó a configurar el movimiento neoconservador (Fried-

3. Para un análisis de algunos de estos aspectos, véase: Whiteley (1992) y Oliver (20|4). 
man, 2005), el cual a su vez puede analizarse como parte de las divisiones y disputas de la sociedad estadounidense de entonces (que han llevado a algunos autores a considerarlas como de guerra civil-Isserman y Kazin, 2000-). O, en igual sentido, podría considerarse la crítica de Bell al hedonismo y consumismo estadounidenses, tema central del libro, como parte de una corriente intelectual más amplia de condena al consumo (Horowitz, 2005).

En este trabajo no se pretende agotar todas estas posibles vías de análisis. Las contradicciones es un libro complejo por los variados temas que aborda y por el tratamiento relativamente especializado que da a cada uno de ellos; no se busca aquí discutir todos ellos. El presente trabajo se centra en las tesis expuestas en la primera parte del libro, que son de carácter más sociológico -y no politológico o económico- y aspiran a ser una lectura más amplia de los problemas del capitalismo. ${ }^{4}$

El interés principal es interrogar qué actualidad se puede atribuir al libro a la luz de la evolución posterior de los fenómenos que analizó y del desarrollo de la teoría social. Se busca ofrecer una lectura que trate de aclarar la vigencia de su diagnóstico sobre las contradicciones del capitalismo y su utilidad como propuesta analítica de las sociedades modernas.

Para lograr este propósito, pareció necesario, primero, perfilar la figura de Daniel Bell como científico social e intelectual público, pues su trayectoria y peso en estos campos son claves de comprensión del texto que se analiza. Después, se entra de lleno al asunto central de Las contradicciones, a saber: su perspectiva sobre las tensiones que enfrenta el capitalismo de su tiempo y el alcance de su diagnóstico. La visión adoptada es crítica. Se quiere mostrar 
cómo su argumento presenta serias debilidades, nacidas de un "estiramiento" injustificado de la tesis weberiana sobre el surgimiento del capitalismo, de un marco analítico que descuida el papel de los sujetos frente a las orientaciones estructurales provenientes de la economía, la cultura y la política, y de una comprensión excesivamente restringida de la cultura.

\section{La figura de Daniel Bell}

Cuando Las contradicciones apareció en 1976, Daniel Bell ya era un académico conocido. Había publicado con anterioridad, entre otros, The end of ideology (1960) y The coming of post-industrial society (1973), dos libros que fueron bien recibidos por los especialistas.

Pero Daniel Bell no escribía sólo para ellos, sino para un público más amplio, interesado en los asuntos públicos. Las contradicciones, y todos sus libros, no surgieron del cubículo o del escritorio, por decirlo así, ni estuvieron ajustados al ritmo lento del trabajo académico; tampoco son trabajos técnicos ni áridos, hechos para la discusión con colegas. Son el fruto, en gran medida, de una exposición de argumentos y tomas de postura dentro de debates públicos, expresados a menudo a modo de ensayos en revistas políticas y culturales. La tesis central de Las contradicciones, por ejemplo, fue inicialmente presentada en un ensayo que lleva ese mismo título y que apareció en el otoño de 1970 en The Public Interest (Bell, 1970), una revista, por cierto, fundada por el propio Bell e Irving Kristol.

Daniel Bell no era, pues, un profesor absorto en la vida académica, aunque recibiera un doctorado por la Universidad de Columbia e hiciera la mayor parte de su carrera académica en Harvard. Fue sin duda lo que llamaríamos un "intelectual público", un hombre de ideas que escribía 
sobre temas de interés general y trataba de incidir en ellos desde cierta perspectiva política o ideológica.

En este sentido, Bell formó parte de un círculo más amplio de intelectuales residentes en Nueva York, de origen judío, varios con antecedentes biográficos y escolares comunes y que lograron gran notoriedad en el clima cultural de la posguerra, como Sidney Hook, Meyer Schapiro, Lionel Trilling, Adam Kazin, Irving Howe, Nathan Glazer y el propio Irving Kristol, entre otros (Bloom, 1986). A varios de ellos se debe la fundación de algunas de las publicaciones periódicas más célebres en Estados Unidos, como The Menorah Journal, Partisan Review, Commentary, Politics, Dissent, Encounter y Public Interest (Jumonville, 2007). Daniel Bell fue un colaborador activo en varias de ellas. Casi todos los ensayos que, reunidos y modificados pasaron a ser capítulos de Las contradicciones, se publicaron con anterioridad en alguna de estas revistas. ${ }^{5}$

Daniel Bell también supo cultivar un rol público desconocido hasta entonces: el del científico social que ofrece descifrar tendencias y avistar los contornos del futuro. Durante la década de los sesenta, Bell formó parte y contribuyó a crear el por entonces naciente campo de lo que se ha dado en llamar con distintos nombres: estudios de futuro, futurología o previsión social.

Fue presidente de una comisión de la Academia Americana de Artes y Ciencias, encargada de hacer un estudio prospectivo con visión al año 2000. Los resultados se publicaron en un número especial de la revista Daedalus en 1967 y luego en forma de libro con algunas actualizaciones posteriores (Bell, 1997). The coming of post-industrial society fue concebido como un ejercicio de esta naturaleza, como su propio subtítulo lo indica (A venture in social forecasting).

5. La faceta de intelectual público de Daniel Bell también tuvo eco en Hispanoamérica con la publicación a partir de 1981 y durante dos décadas de una treintena de sus artículos en la revista Vuelta, dirigida por Octavio Paz (Krauze, 20I I). 
Muchos de sus trabajos tienen una perceptible aspiración en esa línea. Es lo que se advierte también en Las contradicciones: la promesa de ofrecer no sólo un diagnóstico de la situación contemporánea, sino también claves de interpretación del futuro (véase, sobre todo, el capítulo 5).

Bell desempeñaba convincentemente este papel y tenía una distintiva destreza para ello. Aunque es obviamente una licencia poética darle el título de profeta o de "jeremías", las notas periodísticas con motivo de su deceso recordaron sus predicciones sobre la importancia de las computadoras en el siglo XXI y algo que se parece a lo que hoy es la Internet. Hay que acreditarle, por supuesto, la capacidad de colocar en las discusiones académicas temas que luego tendrían una gran resonancia, como el fin de las ideologías, la posmodernidad y la sociedad post-industrial.

Es cierto que esta capacidad de anticipación se debía también a su gran talento para vincular el mundo académico con las preocupaciones del gran público (Waters, 2003, p. 169). Era, sin duda, un gran comunicador. Sabía conectar con una audiencia deseosa de interpretaciones y de orientación práctica. Es significativo que el primero de sus libros y uno de los más famosos, El fin de la ideología, sea en realidad una colección heterogénea de ensayos de los cuales sólo uno, y muy breve, aborda tal cual el presunto fin de las ideologías. Lo mismo se podría decir de Las contradicciones que, como se dijo arriba, fueron en su origen ensayos independientes de los que sólo uno aborda directamente el tema. Que hayan sido los títulos de estos ensayos y no otros los que finalmente aparecerían en la portada de sus libros ilustra su habilidad para cautivar la imaginación del gran público.

Nacido en 1919, Daniel Bell tuvo una vida longeva (murió en 2011, a los noventa y un años). Eso lo hizo testigo de muchos de los acontecimientos decisivos del siglo pasado: el ascenso y caída del Estado de bienestar; las reformas neoliberales de los años ochenta y después; la expansión 
del poderío soviético y su posterior colapso; la revuelta estudiantil de 1968 -de la que fue Bell testigo directo en Columbia y sobre la que editó un volumen colectivo con Irving Kristol (Bell y Kristol, 1969) - y el movimiento contracultural de esos años. Pero además de longeva, tuvo una vida realmente activa. Conferencista incansable, polemista y amante él mismo de las contradicciones, ${ }^{6}$ Daniel Bell es una de las figuras más sui géneris del pensamiento sociológico contemporáneo.

\section{Las contradicciones del capitalismo}

En el epílogo de 1996, Daniel Bell afirma que trató tres temas en su libro de 1976 que expresarían las contradicciones del capitalismo. Estas contradicciones o tensiones son "la tensión entre ascetismo y propensión a adquirir ("acquisitiveness")", "la tensión entre sociedad burguesa y modernismo", y "la separación entre ley y moralidad" (1996, pp. 286 y 287). Efectivamente, esos temas están sugeridos desde las primeras páginas de la edición original, pero sólo los dos primeros son tratados de modo más o menos sistemático a lo largo del libro.

Daniel Bell busca sobre todo explorar el origen, desarrollo y consecuencias de las dos primeras tensiones. Ambas le resultan las principales contradicciones del capitalismo contemporáneo: la entronización del consumo y la subversión de valores impulsada por el modernismo estético. Estas contradicciones sólo pueden entenderse si se les considera en perspectiva histórica.

Bell sostiene que en una época temprana del capitalismo, ya analizada por Max Weber, habría surgido una ética

\footnotetext{
6. En efecto, no podría llamarse sino contradictoria la descripción que hace Daniel Bell de sí mismo en el prefacio de 1978 a su libro Las contradicciones: liberal en política, conservador en cultura y socialista en economía. ¿Se puede ser liberal en política sin serlo, en principio, también en la cultura?
} 
favorable a su expansión, enraizada en el calvinismo como religión y en el puritanismo como modo de vida (1996, introducción y cap. 1). Habría existido durante varios siglos una unidad de orientaciones entre una cultura del ahorro, del esfuerzo y la austeridad, basada en esta ética, y una esfera económica orientada a la ganancia, la acumulación de capital y la eficiencia (1996, especialmente cap. 1, sección II). Por otro lado, la sociedad burguesa tradicional habría mantenido un tipo de experiencia artística y cultural caracterizada por la distancia, la contemplación y los valores estéticos creados en el Renacimiento -la perspectiva en pintura, la narración en la novela y la verdad como correspondencia (1996, pp. 109 y ss.; 1996, pp. 295 y 296)-.

Sin embargo, desde principios del siglo xx comenzó a registrarse un "aflojamiento de los hilos que mantenían juntas la cultura y la economía” (1996, p. Xxx). Frente a sujetos que sacrificaban la gratificación inmediata que su creciente riqueza les permitía lograr y se negaban a llevar una vida ostentosa, a tono con principios religiosos encarnados en prácticas cotidianas, ahora domina una nueva sensibilidad orientada a la adquisición y el placer. Esta transformación de la cultura rompe con la unidad anterior del capitalismo. Mientras que la economía continúa orientándose por la eficiencia y la racionalidad, la cultura lo hace ahora por el hedonismo y se caracteriza por ser "pródiga, promiscua, dominada por un temperamento anti-racional, anti-intelectual, en el que el yo es considerado la piedra de toque de los juicios culturales, y el efecto sobre yo es la medida del valor estético de la experiencia” (Bell, 1996, p. 37).

Esta transformación habría obedecido a un ascenso extraordinario del consumo masivo, provocado por la aparición de las tecnologías domésticas (lavadoras, licuadoras, tostadoras, etc.), el abaratamiento en la fabricación de automóviles, el surgimiento del marketing y, sobre todo, el crédito al consumo (Bell, 1996, pp. 54 y ss.). Combinadas, 
estas invenciones socavaron la ética protestante y el temperamento puritano de sacrificio, ahorro y frugalidad que, en opinión de Bell, fue la matriz cultural de la sociedad norteamericana hasta la década de los cincuenta. Por su parte, el ascenso y consolidación del modernismo cultural disolvió la anterior experiencia estética de la burguesía, en la medida en que destruyó la mímesis como concepto fundamental del arte, trastocó el tiempo convencional de la narrativa e introdujo el experimentalismo como regla (Bell, 1996, pp. 295 y ss.). El modernismo terminó por implantar la idea de que "la vida misma debe ser una obra de arte" (Bell, 1996, pp. 19 y 20).

Esta nueva "sensibilidad cultural" tiene consecuencias sociales más amplias, según Bell, aunque nunca llega a precisarlas. Sus comentarios en este sentido son vagos y generales. Por ejemplo, cuando dice que "llega a ser perturbador para el sistema" ("becomes unsettling to a system") en la medida que implica la pérdida de un "vínculo trascendental" que sí ofrecía la ética protestante y que dotaba a las personas de "significados supremos en su estructura de carácter, su trabajo y su cultura” (Bell, 1996, p. 21). Lo que habría que denunciar, pues, del ascenso de una ética orientada por el placer y basada en el consumo es que "socava la estructura social misma, al golpear al sistema motivacional y de recompensa psíquica que la sustentaba" (Bell, 1996, p. 54). Bell supone que de este modo "el orden social carece de una cultura que sea una expresión simbólica de alguna vitalidad o de un impulso moral que sea fuerza motivacional o vinculatoria. ¿Qué puede mantener unida a la sociedad, entonces?" (Bell, 1996, p. 84).

Daniel Bell construyó su argumento dando por sentado que la ética protestante habría sido, efectivamente, un modo de comportamiento colectivo uniforme y generalizado en la sociedad estadounidense de los primeros siglos (Bell, 1996, p. 21). Es dudoso que haya sido así. El mismo Weber tiene 
claro que su tratamiento de la ética protestante no es una descripción fáctica, sino típica ideal (Weber, 1987).

Bell acepta esto en el epílogo de 1996, al enfatizar que se trataba, en Weber, de una heurística metodológica (1996, p. 289). Sin embargo, esta precaución no se advierte en la primera edición del libro. Bell parece convencido que las pautas morales y de conducta protestante definieron el modo de vida de una sociedad entera a lo largo de toda una época. Es cierto, sin embargo, que la cultura del capitalismo del siglo XVII o del siglo XVIII fue distinta a la del siglo XX en cualquier lugar y aspecto donde se mire.

Mientras que es indiscutible que ha existido un cambio cultural notable a lo largo de los últimos siglos, lo controvertido es si esa transformación de la base moral y cultural del capitalismo puede describirse como un tránsito del ascetismo al hedonismo consumista, como quiere Bell. Ya Werner Sombart (1979) habría mostrado que el consumo superfluo era una práctica común entre las clases ricas desde por lo menos el siglo XVIII, e incluso argumentó que tenía un vínculo digno de consideración con el desarrollo del capitalismo (Franchetti, 2013), una evidencia que el propio Bell tiene en cuenta y discute en el epílogo de 1996.

Aun si se concediera que ese tránsito existió como lo interpreta Bell, no se alcanza a comprender por qué esto significa un problema para la reproducción del capitalismo, afecta el "orden social" o conlleva "inestabilidad en el sistema", cualquier cosa que esto signifique. Es sólo aparente la incompatibilidad entre una economía que requiere comportamientos racionales y cooperativos y una cultura que entroniza el placer y el culto al yo. Como ha señalado Waters (2003), Bell no concede importancia a los incentivos materiales asociados al trabajo y a la participación en la economía. Tampoco parece registrar el hecho de que una característica estructural de las sociedades modernas es la separación entre vida pública y vida privada, con sus 
respectivas bases morales (Bok, 1978; Hampshire, 1978), lo que hace posibles comportamientos, actitudes y valores diferenciados.

Otro problema del argumento de Bell es que parece suponer que el desarrollo y expansión del capitalismo sólo puede ser posible bajo una cultura modelada por el protestantismo, haya o no existido como comportamiento distintivo de toda una sociedad. La ética protestante y su influjo en el capitalismo es, como se sabe, una tesis controvertida, rodeada de malinterpretaciones y debates interminables casi desde que fuera formulada por Max Weber (véase al respecto el monumental estudio de Villegas, 2013).

Weber fue cauto en tratar la relación entre capitalismo y ética protestante como un asunto de "afinidades electivas" entre ambos, y no de causalidad (Weber, 1987; Löwy, 2004), un matiz que no se aprecia en el trabajo de Bell. La evidencia histórica muestra, además, que la relación entre la cultura y/o la religión y la economía capitalista ha sido más compleja de lo que pudo haber entrevisto Weber y parece aceptar Bell (Macfarlane, 1993, caps. VII y VIII; Stone, 1986, cap. VII). ${ }^{7}$ Debe considerarse adicionalmente que el despegue del capitalismo en otras regiones y países no protestantes muestra que existe una pluralidad de culturas y arreglos institucionales que le son compatibles (Hefner, 1998), por lo que no tiene fundamento pensar en el protestantismo como condición única para que suceda.

Más allá de este debate sobre la relación entre ética protestante y capitalismo, Bell es consciente de que la aparición y difusión del consumismo no se debe a impulsos culturales autónomos, sino a la necesidad del capitalismo de crear nuevos mercados (1996, p. 21). Como se señaló antes, Bell enumera varios factores que socavaron la actitud puritana

7. Para documentar la dificultad de analizar el vínculo entre economía y cultura, es de provecho revisar también el trabajo de Rubinstein (1993) en torno a la hipótesis del papel de la cultura en la decadencia económica del Reino Unido.

\section{0}


(si tal cosa sucedió como lo narra), entre ellos el crédito al consumo. En el epílogo, inclusive, desliza la idea de que la base del capitalismo ha cambiado de la producción al consumo (1996, p. 293), una afirmación polémica, por lo demás.

Lo que es significativo, sin embargo, es que estas consideraciones no conducen a Bell a sacar la conclusión debida: que el surgimiento del hedonismo, que parece ser el motor cultural y motivacional de la compra a crédito, no puede ser interpretado como un factor perturbador de, o contradictorio con, la economía, sino, antes bien, como un hecho cultural perfectamente compatible con ella. Bell parece no darse cuenta que el capitalismo es una fuerza avasallante que produce una transformación cultural en sintonía con su propia reproducción. No habría que pensar, entonces, que existe una cultura y una economía que se rigen por imperativos contrarios, como él supone, sino en la emergencia de conductas, valores y expectativas que se complementan recíprocamente en ambas esferas en pos de un mayor avance del capitalismo.

\section{La pérdida de lo sagrado y la necesidad de su restauración}

La insistencia de Bell en el valor perdido de la ética protestante para la funcionalidad del capitalismo, a pesar de los problemas que tiene y que se han buscado poner en evidencia, en realidad esconde un argumento diferente. Bell dedica un capítulo entero de su libro (y menciones significativas en los agregados posteriores) a analizar la religión, las consecuencias que conllevaría su desaparición y la necesidad de restaurar su núcleo fundamental. Esta dedicación es significativa. Muestra que la preocupación de Bell por el ascenso del consumo descontrolado, la satisfacción inmediata y el culto al yo parece radicar menos en su aparente capacidad para lastrar la economía que en su 
poder disolvente de la religión. Según su punto de vista, parece que la ética protestante no sólo era adecuada al desarrollo del capitalismo, sino que era la expresión mundana de convicciones religiosas profundas que ofrecían un sentido trascendental (Bell, 1996, p. 21).

La cultura que reemplazó a la ética protestante no parece ofrecer ese sentido y eso preocupa a Bell, pues parece privar a los individuos de un considerable ingrediente motivacional y de carácter que sí habría sido proporcionado por el protestantismo. Adicionalmente, la cultura moderna operó en sentido inverso a la religión respecto a "lo demoníaco", o sea, frente la naturaleza humana sin control (nature human unchecked). Mientras que la religión le opuso siempre restricciones y limitaciones, el modernismo estético lo liberó y lo convirtió en un valor y en un elemento de creatividad. Sin embargo, aun este desarrollo de una cultura ya secularizada e inmanente, que se expresa a través del arte, ha dejado de tener fuerza. Su impulso se agotó con el debilitamiento de las vanguardias artísticas y su conversión en un estilo de vida de las masas (Bell, 1996, pp. 19, 20 y 29). Lo que hay, entonces, es un vacío de sentido, una "crisis espiritual":

El problema real de la modernidad [itálicas originales] es el de la creencia. Para usar una expresión anticuada, es una crisis espiritual, pues los nuevos asideros han demostrado que son ilusorios y los viejos han quedado sumergidos. Es una situación que nos lleva de vuelta al nihilismo; a falta de un pasado o un futuro, sólo hay un vacío (Bell, 1996, p. 28).

Para arribar a esta conclusión, Bell elabora algunas ideas sobre la cultura y la religión que expone con cierto detalle en el capítulo 4 del libro y que vale la pena revisar brevemente. Lo que se quiere hacer notar aquí son tres problemas: primero, que las definiciones de cultura y religión que elabora son casi sinónimas y, en ambos casos, restringidas a un sentido dramático-existencial; segundo, que los posi- 
bles efectos perjudiciales de un deterioro de la religión se derivan necesariamente de esta visión recortada de ambos conceptos; y tercero, que la insistencia en la necesidad de mantener la distinción entre lo sagrado y lo profano, o de evitar que todo se vuelva profano, si bien se inspira en Durkheim, no tiene en cuenta la convicción durkheimiana de que la religión siempre existirá, aunque asumiendo otras formas.

En cuanto al primer problema, hay que observar que Bell esboza un concepto de cultura que explícitamente se aparta del significado antropológico del término. En su perspectiva, la cultura debe entenderse como un orden simbólico expresivo. Son creaciones que "exploran y expresan los significados de la existencia humana en alguna forma imaginativa" (1996, p. 12). La cultura derivaría de situaciones existenciales que enfrentan todos los seres humanos, como la muerte, el honor, la lealtad, el sacrificio, la obligación, etc.

En el capítulo 4, todo él dedicado a la religión, Bell dice algo semejante respecto a este fenómeno. Junto a la cultura y el trabajo, la religión es un orden simbólico que ofrece significado a los seres humanos para vincularse con el mundo (1996, p. 146). De modo más específico, y como lo precisa en un texto que publicó en 1978, es una especie de recurso cultural que permite a los individuos enfrentar los dilemas existenciales que surgen de su condición de ser "arrojados" en el mundo. Se trata de un conjunto coherente de respuestas ante las preguntas fundamentales de la existencia humana, respuestas que se codifican en ritos, creencias e instituciones (Bell, 1978, p. 34). No es difícil reconocer en esta definición la influencia del existencialismo y el eco que tuvo en la comprensión del hecho religioso en los sociólogos americanos de la década de los sesenta (de igual opinión 
es De la Fuente, 2013). ${ }^{8}$ Una visión de este tipo no es falsa, pero sí limitada: deja de lado los múltiples modos en que la cultura significa y dota de sentido a la vida cotidiana de las personas (De Certeau, 2007).

El segundo problema surge de modo inevitable cuando la cultura y la religión se reducen a ser instrumentos para enfrentar dilemas existenciales: su ausencia se perfila indeseable. Bell formula expresamente el supuesto detrás de esta perspectiva:

La pérdida de significados en estas áreas (cultura, religión, trabajo) crea un conjunto de incomprensiones que la gente no puede soportar y ante lo cual buscan, con carácter de urgente, nuevos significados, para que no sólo quede una sensación de nihilismo o vacío (1996, p. 146).

La plausibilidad del argumento de que el debilitamiento de la religión es un grave problema radica en esta premisa. Los seres humanos necesitan significados religiosos -y culturales y laborales, agregaría Bell- porque, de otro modo, les sería intolerable el posible vacío que sobrevendría. La idea de una experiencia de vacío, de pérdida de significación y de nihilismo es hipotética, no una descripción de un estado de cosas existentes. Surge de una visión filosófica del ser humano, por lo que tiene igual legitimidad que otros modelos de considerarlo (Hollis, 1977). De nuevo, además, aparece el problema de excluir numerosos modos y expresiones en que la cultura y la religión dotan de significación a la vida de las personas.

El tercer problema es apelar a la distinción entre lo sagrado y lo profano sin, a la vez, recuperar la idea de Durkheim de una constante reproducción de lo religioso

8. También para Berger (1999), autor de una de las obras más influyentes de sociología de la religión, por ejemplo, la religión debe considerarse un recurso para enfrentar situaciones-límite (en la connotación de Karl Jaspers) comunes a todos los seres humanos.

\section{4}


bajo nuevas formas. Bell usa la distinción para criticar los empeños transgresores del modernismo que terminan por volver profano lo que antes era sagrado. Desea imponer límites a esta búsqueda de nuevas fronteras expresivas y cree que es necesario reinstaurar o defender ámbitos de sacralidad que considera vitales para el ser humano. Sin embargo, como Durkheim hizo patente, la creación de nuevas expresiones de lo sagrado acompaña a todo proceso de socialidad intensa y duradera, pues es mediante ellas que los grupos se dotan de identidad y permanencia (véase: Durkheim, 2012). No es el caso entonces que haya un déficit de sacralidad en el mundo moderno, sino sólo nuevos modos en que se expresa lo sagrado.

\section{La teoría social de Las contradicciones}

Aun con la mayor generosidad posible es difícil considerar a Daniel Bell un teórico de la sociedad, al menos como se concede que lo son Giddens, Habermas o Bourdieu. Salvo alguna excepción o mención ocasional como tal (Waters, 2003), Daniel Bell no figura entre los teóricos de la sociología. No es sólo que Bell nunca produjo una obra inequívocamente teórica, sino que sus ideas sobre qué es la sociedad, cómo se integra, evoluciona o entra en crisis son demasiado esquemáticas.

Es famosa la autodefinición de Bell como un especialista en generalizaciones, algo que sin duda se advierte en el modo en que observa a las sociedades modernas. Sólo la formulación de generalizaciones -que, por definición, no se detienen en los detalles- podría dar cuenta de procesos cuyo desarrollo abarca decenas o cientos de años. Diremos que Bell está interesado, sobre todo, en una visión a gran escala de las sociedades y de su evolución histórica. En esto se parece a historiadores y sociólogos que propusieron no interpretaciones de acontecimientos, sino de épocas; no lec- 
turas de situaciones, sino de macroprocesos. Como Guizot, Comte, Tocqueville, Marx, Gellner y Toynbee -a quien cita, por cierto, al inicio de The Coming of the post-industrial age-, Bell quiere comprender las transformaciones de gran calado que atraviesan a la sociedad en su conjunto.

La búsqueda de una visión global e histórica por parte de Bell se basó en un marco analítico de las sociedades modernas que supone la distinción entre tres ámbitos: economía, política y cultura. Bell afirma que se trata de tres esferas autónomas, gobernadas cada una por principios diferentes, con ritmos de cambio distintos y que producen igualmente comportamientos diferenciados. La economía (o tecnoeconomía) se rige por la racionalidad de funciones y se orienta a economizar con base en una estructura burocrática y jerárquica; la política es la esfera del poder y la justicia, está gobernada por el principio de legitimidad y su base estructural es la participación política organizada; la cultura, finalmente, como vimos, es una esfera simbólica orientada a expresar y dar sentido a preocupaciones existenciales comunes a la humanidad, como la muerte, el amor, el deber, etc. (Bell, 1996, introducción).

Lo que es significativo mostrar es que Bell no interpreta esta autonomía como resultado de un proceso histórico que define la época moderna, sino como un esquema teórico que, en su opinión, mejora las teorías sociológicas de su tiempo. En su parecer, las visiones de la sociedad existentes son monolíticas, pues asumen que un solo factor es suficiente para comprender el conjunto, como, en su perspectiva, sucede con la teoría marxista de la sociedad, que concede un gran papel a los modos de producción material como principio explicativo central. Es difícil incluir en tales visiones a la teoría de Parsons (1951), a quien Bell nombra, o a la teoría de Habermas, que conoció en su estado embrionario, porque justamente son teorías que suponen la existencia de procesos de diferenciación socio-histórica que dan naci- 
miento a esferas sociales autónomas regidas por lógicas disímiles. Así puede observarse en la teoría de sistemas de Parsons o en la distinción entre sistema y mundo de la vida ofrecida como esbozo en Habermas (1991).

Más allá de la posibilidad -controvertida- de que Bell haya innovado conceptualmente, reconociendo ámbitos diferenciados de integración de la sociedad, queda de manifiesto que trata con ello de poner en evidencia tensiones, paradojas o contradicciones presentes en las sociedades modernas.

En este sentido, el esquema parece fructífero, pues, en efecto, la emergencia de un ámbito político nominalmente integrado por ciudadanos iguales y que procesa democráticamente sus decisiones no se corresponde con el ejercicio de un poder detentado por oligarquías y ejercido centralmente, como tampoco son compatibles las expectativas culturales de consumo ilimitado con la capacidad de la estructura social para satisfacerlas -algo ya visto por todos los teóricos de la anomia- ni con la sustentabilidad del planeta -un tema, por cierto, no tratado por Bell-; ni, al parecer, el cultivo del yo como un valor en sí mismo con la formación de redes de solidaridad orientadas altruísticamente, un aspecto sobre el cual hay que reconocer que hay evidencia que la contradice (Bellah, 1985; Wuthnow, 1996); o bien, una progresiva secularización de la vida cotidiana de las sociedades modernas que, al parecer, deja sin motivaciones genuinas y perdurables a sujetos liberados de lo sagrado.

Si bien estas interpretaciones que se pueden derivar del esquema de Bell son sugerentes, le falta una dimensión de análisis para hacerlas verosímiles: el ámbito de los sujetos y sus decisiones. En efecto, la pieza ausente en la teoría de la sociedad de Bell es un análisis del actor individual y colectivo. Las contradicciones del capitalismo, si bien pueden derivarse y propagarse a nivel sistémico y de estructuras, no por ello producen efectos no mediados sobre los comportamientos de los sujetos, a los que sería ingenuo 
verlos sólo como replicadores pasivos de orientaciones que los avasallan.

Los sujetos tienen capacidad de agencia y reflexividad que los habilitan para posicionarse activamente frente a estos dilemas, tensiones y contradicciones. Ya Merton (1938) habría mostrado cómo para un caso muy similar al de interés de Bell, a saber, la disyunción entre fines culturales y medios sociales para satisfacerlos, es posible que surjan comportamientos anómicos diferenciados que indican, por lo menos, creatividad de la acción y una pluralidad de respuestas colectivas adaptadas a las circunstancias. Del mismo modo Elster (1998) no ha dejado de señalar cómo, frente a situaciones idénticas, no siempre operan los mismos mecanismos causales en las decisiones que toman los sujetos. Habermas (1983), por su parte, criticando este mismo diagnóstico de Bell desde el punto de vista empírico, habría señalado también la emergencia de valores post-materiales en las sociedades contemporáneas y no sólo orientaciones de valor que refuerzan el consumo.

Valga señalar, por último, cómo la seducción por la teoría de Bell impulsa su esquema analítico a un callejón sin salida si con él se quiere elaborar un diagnóstico de una época histórica. En la medida en que con pretensiones teóricas enfatiza la autonomía a cada ámbito respecto a los demás, en esa misma proporción hace surgir inevitablemente la idea de una posible contradicción entre ellos.

De este modo, lo que podría considerarse un hecho contingente y nuevo del capitalismo moderno, a saber, la progresiva separación y posterior tensión surgida entre la economía, la política y la cultura, cosa que aduce Daniel Bell como lectura de un momento histórico, no pueden sino entenderse como normales condiciones estructurales de interacción entre esferas que se han autonomizado unas de otras a través de procesos evolutivos no aclarados por él. Si con fines analíticos se postula una diferenciación y autar- 
quía de estos ámbitos, no se pueden entonces descansar en la semántica de la contradicción las expectativas de una crítica de las sociedades modernas, a menos, claro está, que se anteponga como ideal una condición unitaria donde no había tensiones entre estos ámbitos, o no eran relevantes.

\section{Balance y conclusiones}

Se ha buscado ofrecer una lectura de Las contradicciones que muestre el alcance y vigencia de su argumento principal. Por tratarse de un libro sin duda influyente y emblemático -agregaríamos- de cierto modo de hacer sociología y de concebir a la sociedad, podría ser aún instructivo someterlo de nuevo a análisis, además de indagar si sus páginas siguen ofreciendo estímulo para pensar los problemas del capitalismo contemporáneo.

Del análisis que se ha hecho, se configura una imagen de Daniel Bell más como un intérprete y crítico de la modernidad que como analista de las tensiones que enfrentaba el capitalismo en los años en que lo escribió. Como él mismo lo indica en la introducción, su mira está puesta menos "en los sucesos de la década” y más en las "crisis culturales, más profundas que acechan a las sociedades burguesas y que, a la larga, desvitalizan a un país" (1996, p. 28).

Lo que pretende Bell a final de cuentas no es tanto indicar los problemas que enfrenta la reproducción del capitalismo cuando la política y, sobre todo, la cultura presentan características en apariencia incompatibles, sino mostrar que las transformaciones de la sociedad y la cultura modernas ocurridas durante el siglo veinte, y que van en la dirección de un mayor hedonismo, exaltación del yo y ausencia de lo sagrado, representan un riesgo civilizatorio. Dicho riesgo, como se vio, sólo podrá ser enfrentado en la medida en que se instaure un límite a la erosión de lo sagrado, a la transgresión y a la constante búsqueda expresiva que caracteriza 
a la modernidad. Esta perspectiva sobre la evolución de la cultura moderna y sus efectos coloca a Daniel Bell dentro de un linaje de pensadores y estetas a "contracorriente", por usar este término de Isaiah Berlin, que desde el siglo XVIII se muestran críticos con la ilustración y observan con temor sus efectos corrosivos sobre la tradición y la moral.

¿Pero es sólo Bell un ideólogo conservador que se horroriza ante la expansión de una cultura secular y narcisista? No del todo. Bell fue un intelectual complejo y un buen teórico social (como alguna vez lo calificó Habermas) y sus trabajos tienen todavía aspectos dignos de conservarse y discutirse. Daniel Bell acierta en mostrar que las sociedades contemporáneas están atravesadas por procesos de diferenciación acelerados que no sólo producen una progresiva autonomía de ámbitos antes unificados, sino que pueden producir tensiones y desajustes difíciles de procesar para las instituciones, las estructuras de gobierno y los sujetos que se ven afectados por ellos. Lo que desde cierta tradición sociológica, que va de Durkheim a Luhmann, es tematizado sólo como un hecho neutral y estructurante de las sociedades modernas, a saber, la progresiva diferenciación social, es comprendido por Bell como un desafío de primer orden.

Se debe reconocer también su análisis sobre el origen del consumismo y sobre sus efectos. Si bien su tratamiento termina siendo un juicio de valor a modo de una condena por debilitar la ética protestante, el consumo desenfrenado es un hecho sociológico de gran importancia por sí mismo y hay que acreditarle a Bell ser uno de los primeros sociólogos en llamar la atención sobre él. Más allá de sus consecuencias sobre un orden moral cualquiera que se mire como deseable, el consumismo plantea problemas para la economía mundial (Assadourian, et al., 2010) y para el medio ambiente (Lange y Meier, 2009), y, desde luego, supone profundas transformaciones en la sociedad y la cultura (Bauman, 2007). 
Se puede considerar a Daniel Bell como representante de un tipo de sociología que sitúa su análisis a nivel macro y en el largo plazo. Aunque es difícil concederle el carácter de sociología histórica a su empeño, pues carece del rigor metodológico y la documentación empírica que hacen distintiva a esta tentativa, sus trabajos ofrecen un tipo de aproximación para entender fenómenos que evolucionan de modo lento, pero que producen grandes transformaciones sociales.

La conexión que siempre hizo Bell de estos análisis con audiencias más amplias que las académicas tampoco es un mérito menor. Una sociología que busca ser audible en el debate público de las ideas constituye, sin duda, uno de sus rasgos distintivos desde su creación y hay que considerar a Bell como uno de sus más prominentes -y polémicosexponentes.

Assadourian, E., Amadeo, M., Starke, L., y Worldwatch Bibliografía Institute (20I0). Transforming cultures: from consumerism to sustainability: a Worldwatch Institute report on progress toward a sustainable society. Londres: Norton.

Bauman, Z. (2007). Consuming life. Cambridge-Malden: Polity Press.

Bell, D. (1960). The end of ideology. Glencoe: The free press. (1970). "The Cultural Contradictions of Capitalism". The public interest, (21), 16-43.

- (1996). The cultural contradictions of capitalism. Twentieth anniversary edition. Nueva York: Basic Books. (1997). Towards the year 2000. Work in progress. Massachusetts: The MIT Press.

- y Kristol, I. (1969). Confrontations: The student rebellion and the universities. Nueva York: Basic Books.

Bellah, R. N. (1985). Habits of the heart: individualism and commitment in American life. Berkeley: University of California Press. 
Bibliografía
Bensman, J., y Vidich, A. J. (1972). "The Cultural Contradictions of Daniel Bell". Journal of Aesthetic Education, 6(I/2), 53-65.

Berger, P. (1999). El dosel sagrado. Para una teoría sociológica de la religión. Barcelona: Kairós.

Bloom, A. (1986). Prodigal sons: the New York intellectuals \& their world. Nueva York: Oxford University Press.

Bok, S. (1978). Lying: moral choice in public and private life. Nueva York: Pantheon Books.

Cairncross, F., y Cairncross, A. (eds.). (1992). The Legacy of the golden age: the 1960s and their economic consequences. Londres-Nueva York: Routledge.

Crittenden, B. (1972). "A Demurral to Daniel Bell's The Cultural Contradictions of Capitalism”. Journal of Aesthetic Education, 6(I/2), I39-I 45.

Crozier, M., Huntington, S. P., Watanuki, J., y Trilateral Commission (1975). The crisis of democracy: report on the governability of democracies to the Trilateral Commission. Nueva York: New York University Press.

De Certeau, M. (2007). La invención de lo cotidiano. México: Universidad lberoamericana.

De la Fuente, E. (20I3). “'Profane' rather than 'secular' Daniel Bell as cultural sociologist and critic of modern culture". Thesis Eleven, I I 8(I), I05-I I5.

Durkheim, E. (20I2). Las formas elementales de la vida religiosa. México: Fondo de Cultura Económica.

Elster, J. (1998). “A plea for mechanisms”, en P. Hedstrøm, y R. Swedberg (eds.), Social Mechanisms: An Analytical Approach to Social Theory (pp. 45-73). Cambridge: Cambridge University Press.

Featherstone, M. (199|). Consumer culture and postmodernism. Londres: Sage.

Franchetti, C. (20I3). "A reconsideration of Werner Sombart's Luxury and capitalism”. International Review of Social Sciences and Humanities, 5(2), I35-139. 
Friedman, M. (2005). The neoconservative revolution: Jewish Bibliografía intellectuals and the shaping of public policy. Cambridge: Cambridge University Press.

Habermas, J. (1983). "La ruptura entre cultura y sociedad. Crítica a la cultura de los neoconservadores". Nueva Sociedad, (68), 27-32.

(1991). Problemas de legitimación en el capitalismo tardío. Buenos Aires: Amorrortu.

Hampshire, S. (1978). Public and private morality. CambridgeNueva York: Cambridge University Press.

Hefner, R. W. (1998). Market cultures: society and morality in the new Asian capitalisms. Boulder: Westview Press.

Hollis, M. (1977). Models of man: philosophical thoughts on social action. Nueva York: Cambridge University Press.

Horowitz, D. (2005). Anxieties of affluence: critiques of American consumer culture 1939-1979. Boston: The University of Massachusetts Press.

Illouz, E. (1992). Consuming the romantic utopia. Love and the cultural contradictions of capitalism. Berkeley: University of California Press.

Isserman, M., y Kazin, M. (2000). America divided: the civil war of the 1960s. Nueva York: Oxford University Press. Jumonville, N. (2007). The New York intellectuals reader. Nueva York: Routledge.

Krauze, E. (20II). "Nuestro Jeremías. Daniel Bell (191920I I)". Letras libres, XIII(marzo), 46-48.

Lange, H., y Meier, L. (2009). The new middle classes: globalizing lifestyles, consumerism and environmental concern. Dordrecht-Londres: Springer.

Löwy, M. (2004). "Le concept d'affinité élective chez Max Weber". Archives de sciences sociales des religions, (127), 93- 103.

MacDonald, K. B. (1998). The culture of critique: an evolutionary analysis of Jewish involvement in twentieth-century intellectual and political movements. Westport: Praeger. 
Bibliografía

Macfarlane, A. (1993). La cultura del capitalismo. México: Fondo de Cultura Económica.

Marglin, S. A., y Schor, J. B. (1992). The Golden age of capitalism. Reinterpreting the experience of postwar. Nueva York: Oxford University Press.

Merton, R. K. (1938). "Social Structure and Anomie". American Sociological Review, 3(5), 672-682.

Muñiz, J. N. (1978). "Cultura y crisis en el capitalismo avanzado. Acotaciones en torno a Daniel Bell”. Reis, (I), 283-292.

Offe, C. (1990). Contradicciones en el estado del bienestar. Madrid: Alianza Editorial.

Oliver, P. (2014). Hinduism and the 1960s: the rise of a counter-culture. Nueva York: Bloosmbury.

Parsons, T. (195I). The social system. Glencoe: Free Press. Phillips, G. (1978). "A Commentary on Daniel Bell's Book The Cultural Contradictions of Capitalism". Leonardo, I I (I), 49-5I.

Rubinstein, W. D. (1993). Capitalism, culture, and decline in Britain, 1750-1990. Londres-Nueva York: Routledge.

Sombart, W. (1979). Lujo y capitalismo. Madrid: Alianza Editorial.

Stone, L. (1986). El pasado y el presente. México: Fondo de Cultura Económica.

Touraine, A. (197I). The post-industrial society; tomorrow's social history: classes, conflicts and culture in the programmed society. Nueva York: Random House.

Yates, J., y Hunter, J. D. (20I I). Thrift and thriving in America. Capitalism and Moral Order from the Puritans to the Present. Nueva York: The Oxford University Press.

Villegas, F. G. (20I3). Max Weber y la guerra académica de los cien años. La polémica en torno a La ética protestante y el espíritu del capitalismo (I905-20/2). México: Fondo de Cultura Económica. 
Waters, M. (2003). “Daniel Bell”, en G. Ritzer (cood.), The Bibliografía Blackwell companion to major classical social theorists (pp. 154- 177). Malden: Blackwell.

Weber, M. (1987). "La ética protestante y el espíritu del capitalismo", en, Ensayos sobre sociología de la religión. Tomo I (pp. 25-232). Madrid: Taurus.

Whiteley, S. (1992). The space between the notes: rock and the counter-culture. Londres-Nueva York: Routledge.

Wuthnow, R. (1996). Actos de compasión: cuidar de los demás y ayudarnos a nosotros mismos. Madrid: Alianza. 